\title{
Different Surgical Approach To Idiopathic Gingival Enlargement: A Case Report
}

\author{
Maj Anupam Gupta, COL S. K. Rath \\ Dental Officer, 9 CDU, Dharmashala, Himachal Pradesh, India \\ E-mail for correspondence: anupam12cods@gmail.com
}

\begin{abstract}
"Gingival enlargement" is the increase in the size of gingiva which usually overfills the interproximal spaces, ballooning out over the teeth and sometimes even protruding into the oral cavity. It may occur as a localized enlargement or may be generalized involving the entire dentition. It is multifactorial in origin having the influence of bacterial plaque, systemic factors, genetic predisposition, and various medications. Sometimes, it may be idiopathic without any specific etiologic factor. Two different treatment modalities, namely scalpel and electrocautery can be used for treating gingival enlargement. In the present case report, these two different treatment modalities were used in the same patient with idiopathic gingival enlargement to compare their handling properties and effect on initial wound healing.
\end{abstract}

Key words: Electrocautery, enlargement, gingiva, gingivectomy

\section{INTRODUCTION}

Increase in size of the gingiva is a common feature of the gingival disease and is termed as gingival enlargement. Generalized gingival enlargement is characterized by massive and exuberant gingival overgrowth that appears to involve and cover the tooth surfaces throughout the mouth. ${ }^{[1]}$ It can be due to various causes. Most prevalent are the inflammatory, idiopathic, hereditary gingival fibromatosis, drug-induced, and systemic diseases or conditions, for example, diabetes mellitus and leukemia. ${ }^{[2,3]}$

Hereditary gingival fibromatosis is a slowgrowing gingival enlargement with great clinical and genetic heterogeneity. The enlargement affects the attached gingiva, as well as gingival margin and interdental papillae in contrast to drug-induced overgrowth, which is often limited to gingival margin

\begin{tabular}{|c|c|}
\hline Quick Response Code & Article Info: \\
\hline & doi: $10.5866 / 2018.10 .10087$ \\
\hline & $\begin{array}{l}\text { Received: } 16-04-2018 \\
\text { Revised: } 18-05-2018 \\
\text { Accepted: } 12-06-2018 \\
\text { Available Online: } 15-07-2018,2018 \\
\text { (www.nacd.in)@ NAD, } 2018 \text { - All rights } \\
\text { reserved }\end{array}$ \\
\hline
\end{tabular}

and inter-dental papillae. The enlarged gingiva is pink, firm, and leathery in consistency and has a pebbled or stippled surface, with little tendency to bleed. ${ }^{[4]}$ In severe cases, it leads to overwhelming gingival tissue growth completely covering the teeth and projects into the oral vestibule.

Diagnosis of various forms of gingival enlargements is substantiated by the patient's history, clinical evaluation, panoramic radiography, and histopathological examination. ${ }^{[5]}$ The definitive treatment is surgical intervention and good oral hygiene maintenance to improve the compromised esthetics and functions. Here is an interesting case report of idiopathic gingival enlargement persisting up to adult stage and being treated by the dual approach, one side with manual surgery and the other with the electrosurgical approach.

\section{CASE REPORT}

A 26-year-old female patient reported to the Department of Dental Surgery at 9 Corps Dental Unit with a chief complaint of swollen gums involving all her teeth since past 3 years preventing proper speech, mastication, and poor esthetics. She did not give any history of drug intake, fever, anorexia, weight loss, seizures, hearing loss, or mental disorder. Familial and postnatal history was non-contributory. 
Extra-oral examination revealed that the patient has no abnormality. An intraoral examination revealed generalized, diffused, nodular enlargement of the gingiva involving the upper and lower arches, which were pink in color, and had a firm and fibrous consistency [Figure 1]. The teeth were barely visible as the enlarged gingiva covered the incisal/occlusal third of the teeth. Radiological investigations revealed no bone loss [Figure 2]. Hematological investigations were within normal limits.

Punch biopsy carried out revealed bulbous increase in the connective tissue, which was relatively avascular and had densely arranged collagen-fiber bundles, numerous fibroblasts, and mild chronic inflammatory cells. The overlying epithelium exhibited hyperplasia and had elongated rete ridges suggesting the histological diagnosis of fibroepithelial hyperplasia [Figure 3].

After completion of Phase I, a quadrant-wise gingivectomy was performed under local anesthesia

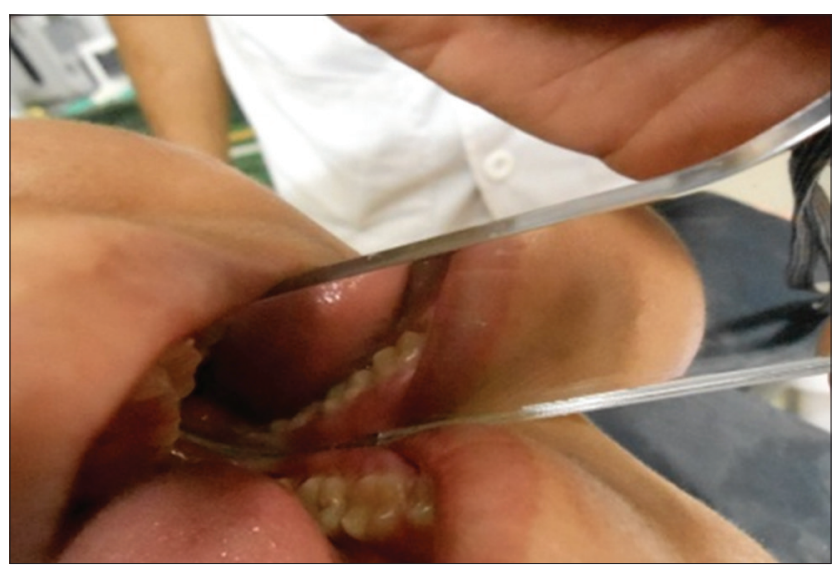

Figure 1: An intraoral examination reveals generalized, diffused, and nodular enlargement of the gingiva involving the upper and lower arches

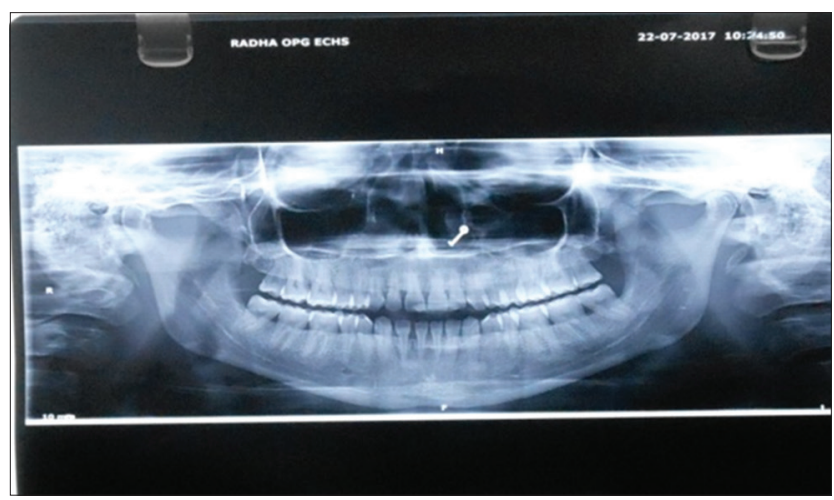

Figure 2: Orthopantomogram showing no bone loss using two different techniques. In the third and fourth quadrants, scalpel technique was performed [Figure 4]. Kirkland knife was used for incisions on the facial and lingual surfaces. Orban periodontal knives were used for interdental incisions. External bevel gingivectomy was done. 10 days later, surgery using an electrocautery device with a medium size, loop electrode was performed in the first and second quadrant [Figure 5]. Coe-pack was given in both quadrants to reduce patient discomfort. Use of electrocautery provided excellent hemostasis and better immediate post-operative result. Furthermore, the patient acceptance toward the

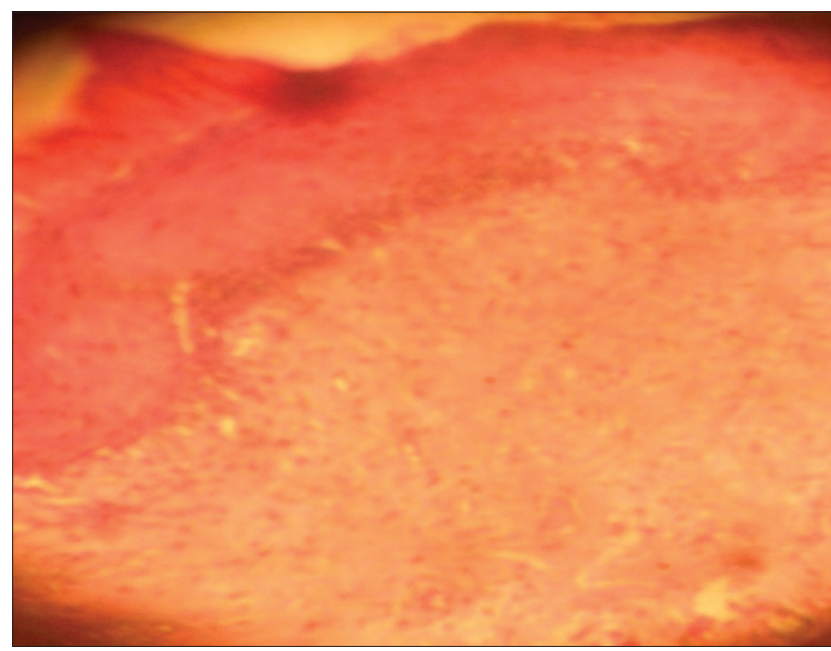

Figure 3: $\mathrm{H}$ and $\mathrm{E}$ section showing densely arranged collagen-fiber bundles, numerous fibroblasts, and mild chronic inflammatory cells. The overlying epithelium exhibits hyperplasia and has elongated rete ridges suggesting the histological diagnosis of fibroepithelial hyperplasia

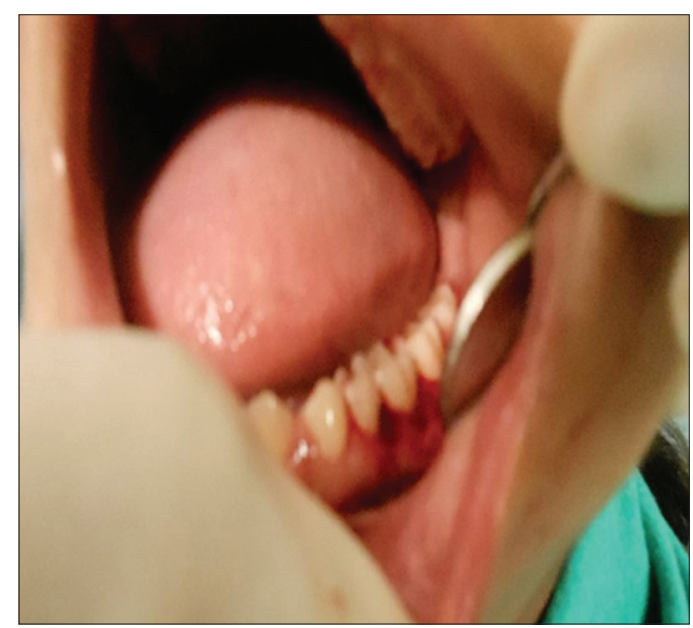

Figure 4: Clinical picture showing the scalpel technique in the third and fourth quadrants 
treatment was better with these two rather than the conventional approach [Figure 6].

\section{DISCUSSION}

Idiopathic fibrous enlargement of gingiva may be congenital or hereditary. The majority of reported cases have been attributed to hereditary factors, the mode of transmission mainly being autosomal dominant. ${ }^{[6,7]}$ Many cases are sporadic with no familial background; however, longterm use of drugs has to be ruled out during case history taking. A comprehensive medical history and physical systemic evaluation are essential to rule out other systemic abnormalities. Gingival hyperplasia may also be associated with physical development, retardation, and hypertrichosis. ${ }^{[8]}$ Although gingival tissue may appear normal at birth, hyperplasia of the gingiva may become evident with the eruption of primary or permanent dentition, suggesting a trauma-induced tissue reaction during the eruption. ${ }^{[9]}$ Further enlargement does not occur once; the growth of the jaws is completed. ${ }^{[10]}$ Gingival enlargement may also be due to nutritional and hormonal factors; however, these have not been completely substantiated.

The constant increase in the tissue mass can result in delayed eruption and displacement of teeth, arch deformity, spacing, and migration of teeth. ${ }^{[11]}$ Due to massive gingival enlargement, an affected child usually develops abnormal swallowing

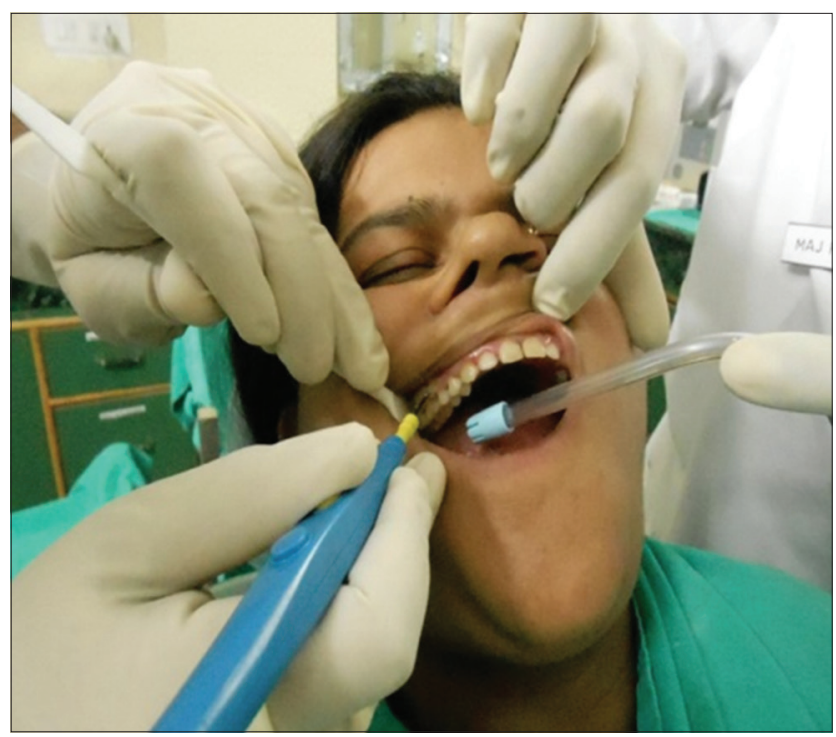

Figure 5: Clinical picture showing surgery using an electrocautery device with a medium size, loop electrode in the first and second quadrant pattern and experiences difficulty in speech and mastication. In the present case, the enlarged gingival tissue partially covered the occlusal surface of the molars, getting traumatized during mastication and causing pain. Along with this, there was some interference with oral hygiene measures and normal mastication. This favors accumulation of plaque, which further complicates the existing hyperplastic tissue. Maintenance of good oral hygiene is very important. It is not known if plaque control measures are effective in this condition; however, it is a good practice to maintain the plaque control following gingivectomy procedure.

Various modalities of treatment have been proposed including radical treatment with extraction of the involved teeth, which was reported not to favor a recurrence of the growth. Two most favored treatment modalities being scalpel and electrocautery. In the present case report, these two different treatment modalities were used in the same patient with idiopathic gingival enlargement to compare their handling properties and effect on the initial wound healing. In the quadrants where scalpel was used, the surgical procedure was complicated with excessive hemorrhage even though the tissue appeared pale and firm. The other disadvantages of scalpel being need of anesthesia, inadequate visibility caused by blood in the operating field, non-sterilized incision cut. On the other hand, the advantages observed were ease of use, precise incision with well-defined margins, relatively fast uneventful healing, and no unwanted lateral tissue damage. When electrocautery was used, cuts were made with ease, since the electrode cuts on its side

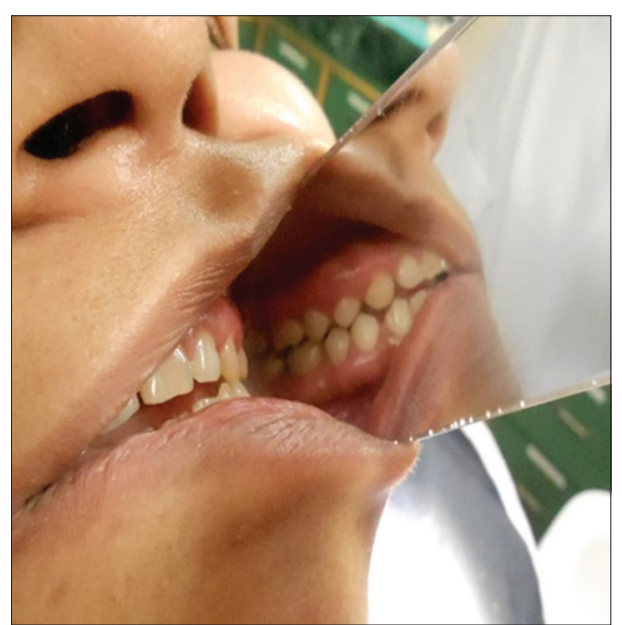

Figure 6: Clinical picture showing complete healing and better results than the conventional approach 
as well as on its tip. Hemostasis is immediate and consistent, the wound is nearly painless, and the tip is self-disinfecting. Disadvantages observed were need of anesthetic agent before cutting, unavoidable burning-flesh odour, low tactile sense, does not allow for their use around implants, bone can be damaged and can be dangerous in an explosive environment. Furthermore, electrocautery is contraindicated in patients with pacemakers, poor post-operative healing who have undergone irradiation, diabetes, or blood dyscrasias.

Since recurrence could be expected within a few months after surgery and the gingiva may return to the original condition within a few years, the patient may have to undergo repeated gingivectomy procedures. This often causes a further increase in the patients' and parents' psychological and emotional stress. Hence, psychological counseling was recommended for the patients and parents.

\section{CONCLUSION}

Electrosurgical excision is an excellent, easily available, and economical surgical technique for the management of generalized gingival enlargement. Genetic consultation is mandatory for future family planning in case of hereditary gingival enlargement. Education and awareness regarding the special conditions such as puberty, pregnancy, medications as well as meticulous oral hygiene measures, and early referral to tertiary care centres are essential to reduce the occurrence and proper management of gingival enlargement. Moreover, cross-comparative studies are required to establish some diagnostic and therapeutic standards for such patients.

\section{REFERENCES}

1. Newman MG, Takei HH, Klokkevold PR, Carranza FA. Carranza's Clinical Periodontology. Gingivalenla-Rgement. $10^{\text {th }}$ ed. Philadelphia: Elsevier Publishers; 2006. p. 741-77.

2. Babu KB, Pavankumar K, Anuradha BR, Arora N. Hereditary gingival fibromatosis - A case report and management using a novel surgical technique. South Brazil Dent J 2011;8:453-8.

3. Tomar N, Vidhi M, Mayur K. Inflammatory gingival enlargement-a case report. J Adv Med Dent Sci 2014;2:109-13.

4. Katz J, Guelmann M, Barak S. Hereditary gingival fibromatosis with distinct dental, skeletal and developmental abnormalities. Pediatr Dent 2002;24:253-6.

5. Pushpanshu K, Kaushik R, Sathawane RS, Athawale RP. Extensive gingival enlargement in siblings. Sultan Qaboos Univ Med J 2012;12:517-21.

6. Cortelli JR. Evidence of genetic heterogenecity for hereditary gingival fibromatosis. J Dent Res 2000;79:1758-64.

7. Hart TC, Pallos D, Bowden DW, Bolyard J, Pettenati MJ, Cortelli JR. Linkage of hereditary gingival fibromatosis to chromosome 2 21. Am J Hum Genet 1998;62:876-83.

8. Shafer WG, Hine MK, Levy BM. Developmental Disturbances of the Perioral Structures. $4^{\text {th }}$ ed. Philadelphia, PA: A Prisma Indian; 1993. p. 23-4.

9. Gupta N, Maheshwari S. Advanced gingival fibromatosis. J Indian Dent Assoc 1996;167:46-7.

10. Stewart RE. Periodontal diseases in children. In: Pediatric Dentistry and Clinical Practice. USA: Mosby Company; 1982

11. Mcdonald RE, Avery DR. Gingival and periodontal diseases. In: Dentistry for the Child and Adolescent. $7^{\text {th }}$ ed. USA: Mosby Company; 2000 